\title{
Engineering Metallic Nanocavity Radiation for Efficient Uni-/Bi-directional Coupling into Integrated Waveguide
}

\author{
Myung-Ki Kim ${ }^{1^{*}}$, Zheng $\mathrm{Li}^{1}$, Ryan Going ${ }^{2}$, Ming C. $\mathrm{Wu}^{2}$, and Hyuck $\mathrm{Choo}^{{ }^{*}}$ \\ ${ }^{1}$ The Moore Laboratory, Electrical Engineering, California Institute of Technology, Pasadena, CA 91125 \\ ${ }^{2}$ Electrical Engineering \& Computer Sciences, Cory Hall, UC Berkeley, Berkeley, CA 94720 \\ *mkkim@caltech.edu,hchoo@caltech.edu
}

\begin{abstract}
We propose a new, simple way to engineer the radiation patterns of subwavelengthscale metallic semiconductor cavities for coupling light from a nanoscale metal cavity into integrated waveguides uni-/bi-directionally with efficiency up to $\sim 90 \%$.

OCIS codes: (250.5403) Plasmonics; (230.5750) Resonators; (250.5300) Photonic Integrated Circuit
\end{abstract}

\section{Introduction}

One of the most promising ways to improve the performance of microscale optical communication components is to reduce their volumes down to the nanoscale while preserving their desirable optical properties in the microscale. From this viewpoint, metallic subwavelength-cavities can be very attractive because they provide both very small physical volumes and high quality factors (Q-factors) [1]. However, the far-field radiation patterns of the subwavelength-cavities are intrinsically very broad due to their small output apertures, making it difficult to couple the light into integrated waveguides [2]. In this work, we propose a novel clad-engineering method that allows us to control the far-field radiation patterns of the subwavelength-scale metallic semiconductor cavity and efficiently couple light into the integrated waveguide uni-/bi-directionally, with efficiency almost up to $90 \%$.

\section{Radiation engineering in metallic semiconductor nanocavity}

A metallic semiconductor nanocavity is shown in Fig. 1(a). The structure is composed of 1) a subwavelength-scale InGaAsP nanocavity, 2) a low refractive index $\mathrm{SiO}_{2}$ cladding layer that wraps around the cavity, and 3) the outermost silver layer that surrounds the $\mathrm{SiO}_{2}$ layer, except the bottom surface of the structure. Decreasing the cladding thicknesses $\left(a, b_{1}, b_{2}\right.$ defined in Fig. 1(a)) at the same rate (along the $\mathrm{x}$-/y-directions or the major wavevector directions of the cavity mode) will greatly increase the Q-factor and also preserve the original fundamental TE-mode profile (Fig. 1(b)). However, having $90^{\circ}$ rotational symmetry, the near field distribution of the fundamental TE mode spreads out radially along the $\mathrm{x}$-/y-directions, and its far-field radiation pattern becomes very broad in the $\mathrm{x}-\mathrm{y}$ plane near the bottom of the structure (Fig. 1(e)). And, this makes coupling the radiation into another optical component very inefficient.

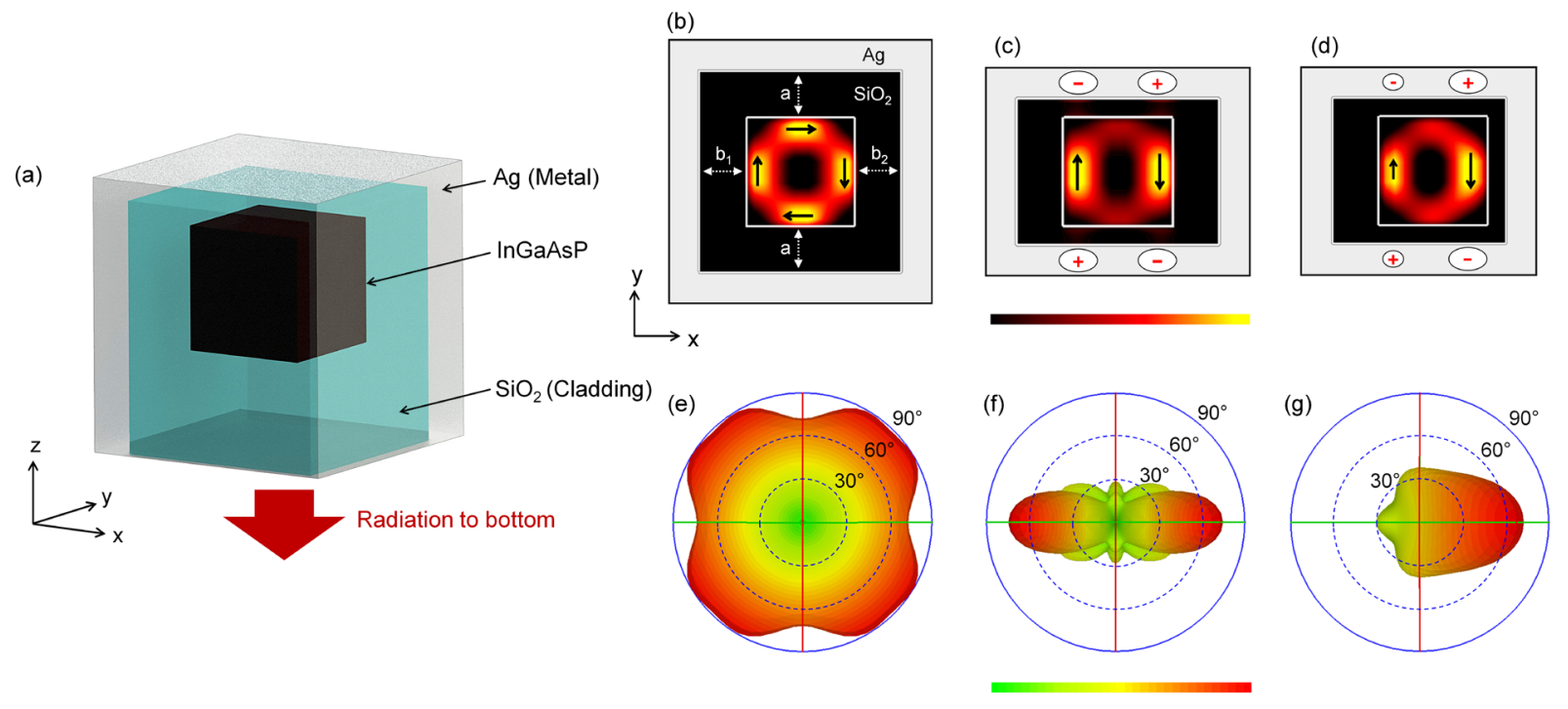

Fig. 1. (a) Schematic illustration of simplified metallic semiconductor nanocavity, where the semiconductor (InGaAs) cavity has the dimensions of $350 \mathrm{~nm} \times 350 \mathrm{~nm} \times 350 \mathrm{~nm}$. Energy-density distributions (overlaid major electric-fields) and the corresponding far-field radiation patterns to the bottom direction (b, e) when $a=b_{1}=b_{2}=150 \mathrm{~nm},(\mathrm{c}, \mathrm{f})$ when $a=60 \mathrm{~nm}, b_{1}=b_{2}=150 \mathrm{~nm}$, and (d, g) when $a=60 \mathrm{~nm}$, $b_{1}=150 \mathrm{~nm}, b_{2}=60 \mathrm{~nm}$, respectively. 
To overcome this inefficiency, we adjust the dimensions of the cladding layers along $\mathrm{x}$-/y-directions. We break the rotational symmetry of the cavity structure, so we could control the far-field radiation patterns, as shown in Fig. 1(c) $\left(a<b_{1}=b_{2}\right.$, for bi-directional radiation) and $1(\mathrm{~d})\left(b_{1}<b_{2}\right.$ for uni-directional radiation). Figure 1(f) and (g) clearly show that the far-field radiation patterns are controlled by adjusting the dimensions of the cladding layer. These highly directional radiation patterns originate from the asymmetric SPP-coupling strengths between the semiconductor cavity and the metal layer due to different intermediate cladding thicknesses.

\section{Efficient waveguide coupling in III-V/Si integration}

The clad engineering method can be immediately applied to accomplish efficient cavity-to-waveguide coupling. Light coupling from III-V nanocavities to single mode silicon-on-insulator waveguides is of particular interest because it provides an easy, effective way to integrate nanoscale light sources, modulators, and detectors for III-V/Si integrated photonics. Figure 2(a) shows the case in which $a<b_{1}=b_{2}$. Coupling efficiency to the bi-directional Siwaveguide increases as the cladding dimension $a$ is reduced. The efficiency increases up to $80 \%$ when $a=40 \mathrm{~nm}$, and the field intensity profile in Fig. 2(b) clearly show that light is efficiently and bi-directionally coupled into the waveguide. Similarly, for $b_{1}<b_{2}$ with $a$ fixed at $80 \mathrm{~nm}$, the coupling efficiency into the unidirectional Si-waveguide increases up to 90\%, as shown in Fig. 2(c), and the field intensity profile in Fig. 2(d) also clearly shows efficient coupling into the integrated waveguide. The degradations of Q-factor in Fig. 2(a) and (c) are attributed to the enhanced cavity-to-waveguide coupling rate as well as some increase in absorption loss in thinner cladding regions.

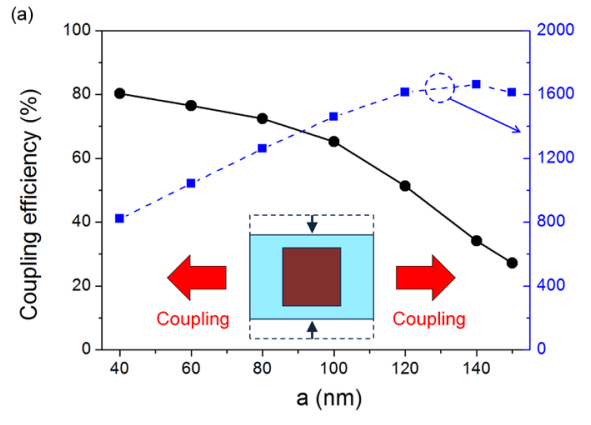

(c)

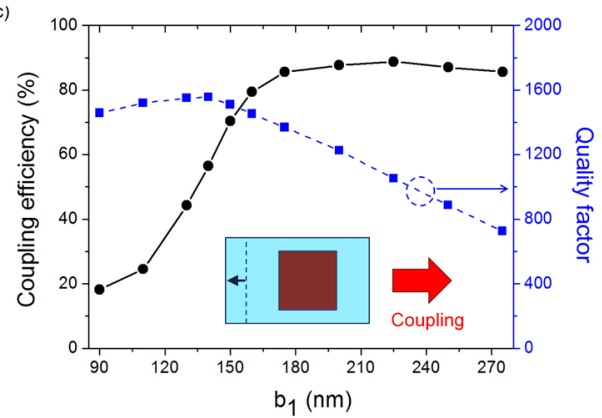

(b)

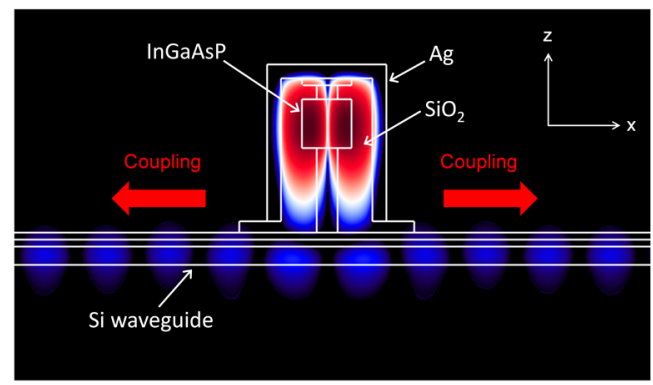

(d)

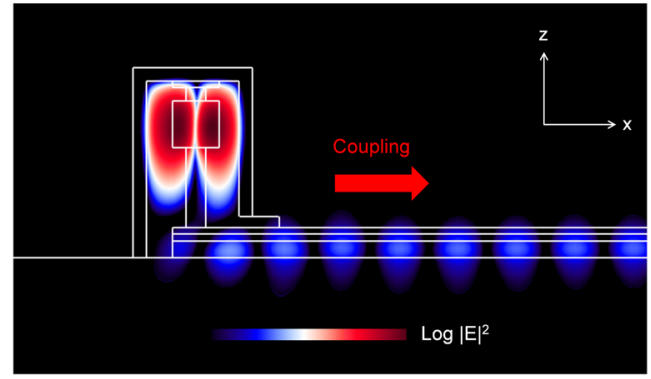

Fig. 2. (a) Coupling efficiency and Q-factor as a function of $a$ in the metallic cavity coupled with bidirectional Si-waveguide. Here, both $b_{1}$ and $b_{2}$ are fixed at $150 \mathrm{~nm}$. (b) Electric-field intensity profile in logarithm scale: metallic cavity mode coupled with bi-directional waveguide, where $a, b_{1}$ and $b_{2}$ are $40 \mathrm{~nm}, 150 \mathrm{~nm}$ and $150 \mathrm{~nm}$, respectively. (c) Coupling efficiency for unidirectional Si-waveguide and Q-factor as a function of $b_{1}$. Here, $a$ and $b_{2}$ are fixed at $80 \mathrm{~nm}$ and $150 \mathrm{~nm}$, respectively. (d) Electric-field intensity profile in logarithm scale: metallic cavity mode coupled with uni-directional waveguide, where $a, b_{1}$ and $b_{2}$ are $80 \mathrm{~nm}, 180 \mathrm{~nm}$ and $150 \mathrm{~nm}$, respectively.

\section{Conclusion}

Using simulations, we have shown that the cladding engineering method is able to control the radiation patterns of the subwavelength-scale metallic semiconductor cavity and accomplish highly efficient cavity-to-waveguide coupling, which would open up new possibilities for realizing on-chip, ultra-compact, extremely fast, and energyefficient III-V/Si-integrated optical communication components.

\section{References}

[1] K. Ding and C. Z. Ning, "Metallic subwavelength-cavity semiconductor nanolasers,” Light: Sci. Appl. 1, e20; 10.1038/1sa.2012.20 (2012).

[2] M.-K. Kim et al., "Efficient waveguide-coupling of metal-clad nanolaser cavities," Opt. Express 19, 23504-23512 (2011). 